\title{
A GEM-TPC for the CBELSA/TAPS experiment
}

\section{Jonathan Ottnad*}

Universität Bonn, Helmholtz-Institut für Strahlen- und Kernphysik, Bonn, Germany

E-mail: jottnad@hiskp.uni-bonn.de

\section{Markus Ball}

Universität Bonn, Helmholtz-Institut für Strahlen- und Kernphysik, Bonn, Germany

\section{Reinhard Beck}

Universität Bonn, Helmholtz-Institut für Strahlen- und Kernphysik, Bonn, Germany

\section{Bernhard Ketzer}

Universität Bonn, Helmholtz-Institut für Strahlen- und Kernphysik, Bonn, Germany

\section{Viktor Ratza}

Universität Bonn, Helmholtz-Institut für Strahlen- und Kernphysik, Bonn, Germany

\section{Dimitri Schaab}

Universität Bonn, Helmholtz-Institut für Strahlen- und Kernphysik, Bonn, Germany

\section{Roman Schmitz}

Universität Bonn, Helmholtz-Institut für Strahlen- und Kernphysik, Bonn, Germany

\begin{abstract}
In order to enhance the sensitivity of the detector system of the CBELSA/TAPS experiment to charged particles, a Time Projection Chamber (TPC) is foreseen as a new tracking detector, which provides track and vertex reconstruction for charged particles. The amplificational stage of the TPC operates with Gas Electron Multipliers (GEMs) [1]. Detailed ANSYS and Garfield++ simulations were carried out and compared to measurements to find the optimum operation point of a complex multi-GEM amplification system.
\end{abstract}

5th International Conference on Micro-Pattern Gas Detectors (MPGD2017)

22-26 May, 2017

Philadelphia, USA

\footnotetext{
*Speaker.
} 


\section{Introduction}

The CBELSA/TAPS experiment located at the Elektronen Stretcher Anlage (ELSA) in Bonn (Germany) investigates the excitation spectrum of baryons and the properties of baryon resonances. The measurements shed light on the dynamics of QCD in the non-pertubative regime.

As depicted in figure 1, the main component of the setup is the Crystal Barrel, a high resolution electromagnetic calorimeter, which is made out of $1320 \mathrm{CsI}(\mathrm{TI})$-crystals. It is optimized for the study of baryonic resonances with neutral final states [2], for example $\gamma p \rightarrow p \pi^{0}, \gamma p \rightarrow p \eta$, $\gamma p \rightarrow p \eta^{\prime}$. The nearly totally absorbing calorimeter covers the full azimuthal angle and the polar angle from $1^{\circ}$ to $156^{\circ}$, making this experiment an ideal candidate to measure a large set of polarization variables in reactions created by an linearly or cirularly polarized photon beam hitting a longitudinal or transversely polarized target $[3,4]$.

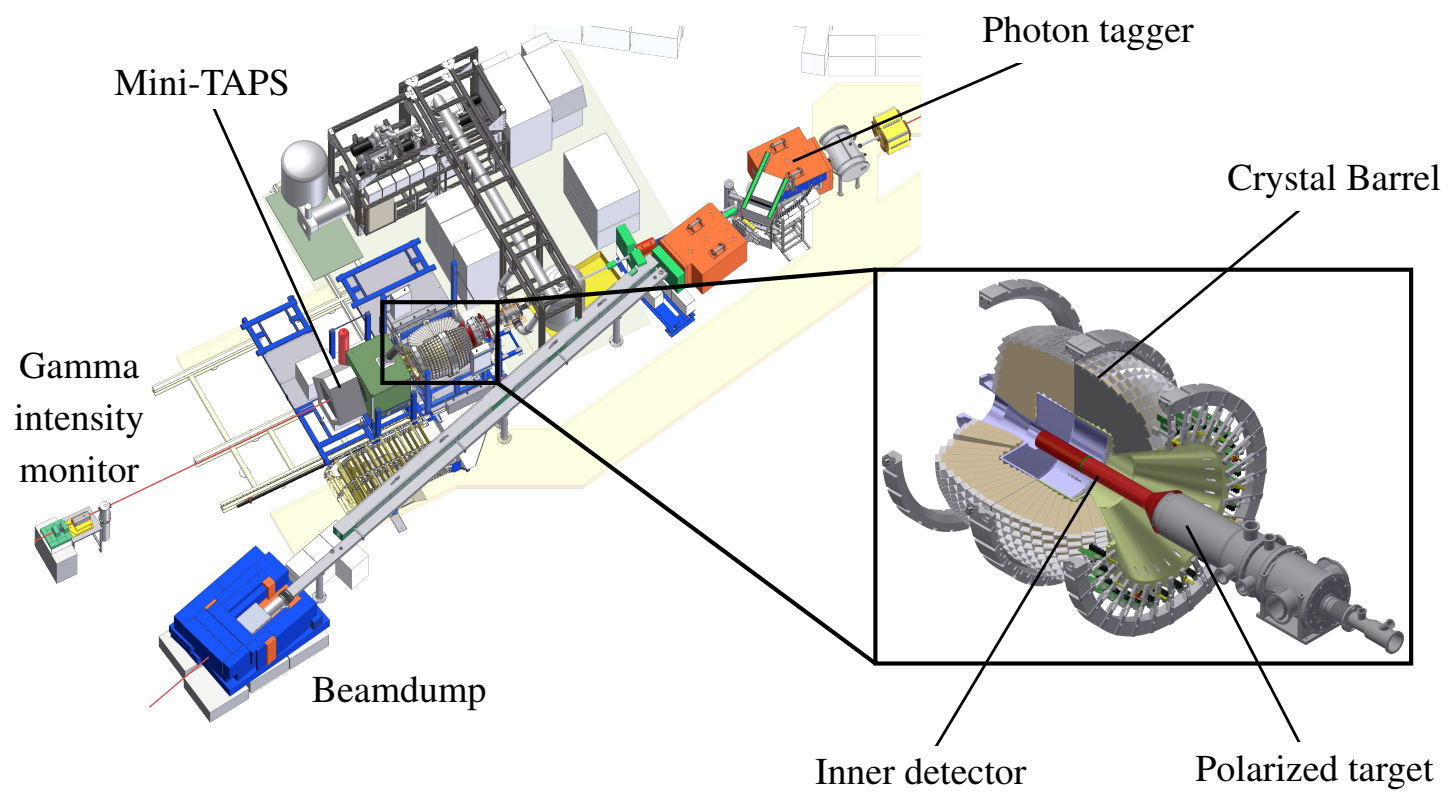

Figure 1: Experimental area of the CBELSA/TAPS setup.

However, the existing setup is optimised, but also restricted, to study reactions with neutral final states. Charged final states are currently only identified by an inner detector of scintillating fibers. Access to charged final states would significantly increase the number of observable events. While for example the neutral decay of the $\omega$-meson $\gamma n \rightarrow n \omega \rightarrow n \pi^{0} \gamma$ has a branching ratio of $B R=8.28 \%$, the charged counterpart $\gamma n \rightarrow n \omega \rightarrow n \pi^{+} \pi^{-} \pi^{0}$ yields a branching ratio of $B R=89.2 \%$ [5]. These reactions with charged final states could be observed with the implementation of a TPC as a new inner tracking detector. A TPC is sensitive to charged particles, offering very precise tracking and vertexing capabilities and, if operated inside a magnetic field, can also determine the specific energy loss. The operation principle and performance of the TPC prototype foreseen for the CBELSA/TAPS experiment will be discussed in the following. 


\section{The TPC prototype}

The TPC (see figure 2) which is foreseen for the CBELSA/TAPS experiment features a cylindrical field cage, with an inner diameter of $104.0 \mathrm{~mm}$, an outer diameter of $308.0 \mathrm{~mm}$, and a drift length of $727.8 \mathrm{~mm}$. Due to the fixed target geometry of the experiment, the TPC is read out single-sided by more than 10000 pads connected to 168 T2K AFTER chips [6]. The gaseous amplificational stage features a stack of GEMs. With an overall material budget of $<1 \% X_{0}$, the TPC hardly affects the outer detectors. Furthermore it covers a polar angle from $10.5^{\circ}$ to $163.0^{\circ}$, which is close to the coverage of the experiments present detector setup.

The TPC prototype was successfully operated at the FOPI spectrometer at GSI, Germany. It observed reactions created by an $1.7 \mathrm{GeV} / c$ pion beam impinging on a carbon target. The detector showed overall good performance, which is reflected in a vertext resolution of $\left\langle\sigma_{X Y}\right\rangle=(270.2 \pm 2.6) \mu \mathrm{m}$ and $\left\langle\sigma_{Z}\right\rangle=(580.3 \pm 5.6) \mu \mathrm{m}$, and an energy resolution of $R=24.5 \% E^{-\frac{1}{2}}$ [7]. As the detector was operated inside a magnetic field, the specific energy loss could be measured as a function of the particle momentum. Figure 3 shows clear bands for pions, kaons, protons, and deuterons. Yielding a $\mathrm{d} E / \mathrm{d} x$ resolution of $14 \%$, this underlines the particle identification capabilities of the TPC.

During the operation of the TPC it was determined, that the next fieldcage will have to improve on several construction flaws. For example the inner fieldcage is not perfectly aligned with respect to the outer fieldcage, resulting in an relative offset of the fieldstrips. Furthermore, sparks between the

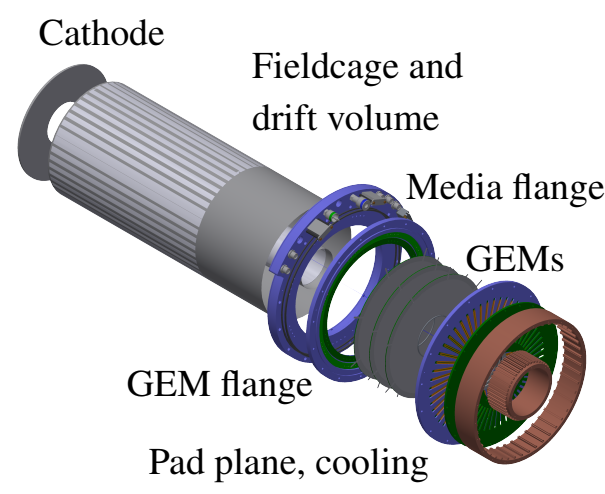

Figure 2: Exploded view of the TPC prototype, featuring a triple GEM stack.

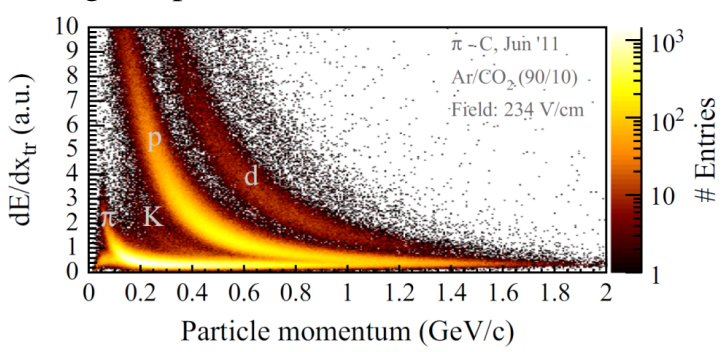

Figure 3: TPC measurement of specific energy loss [8].

cathode and the outer fieldcage caused heavy damage to the fieldcage. Even though the primary damage could be repaired, a couple of fieldstrips remain permanently shortened, resulting in distortions of the drift field. More detailed information on the field distortions can be found in [7].

\section{Optimisation of the GEM-stage}

The main reason to use GEMs in the amplificational stage of a TPC is their capability to be operated ungated even at high particle rates. However, in an ungated mode of operation, ions created in the amplficational stage can unrestrictedly reach the drift region. There they will remain as space charges which produce time dependent, non static field distortions. This poses a tremendous challenge to calibtration methods in order to include these distortions. It is necessary to keep the 
ion backflow at a managable size. This can be achieved by either preventing the ions from drifting back or simply producing less ions in the beginning.
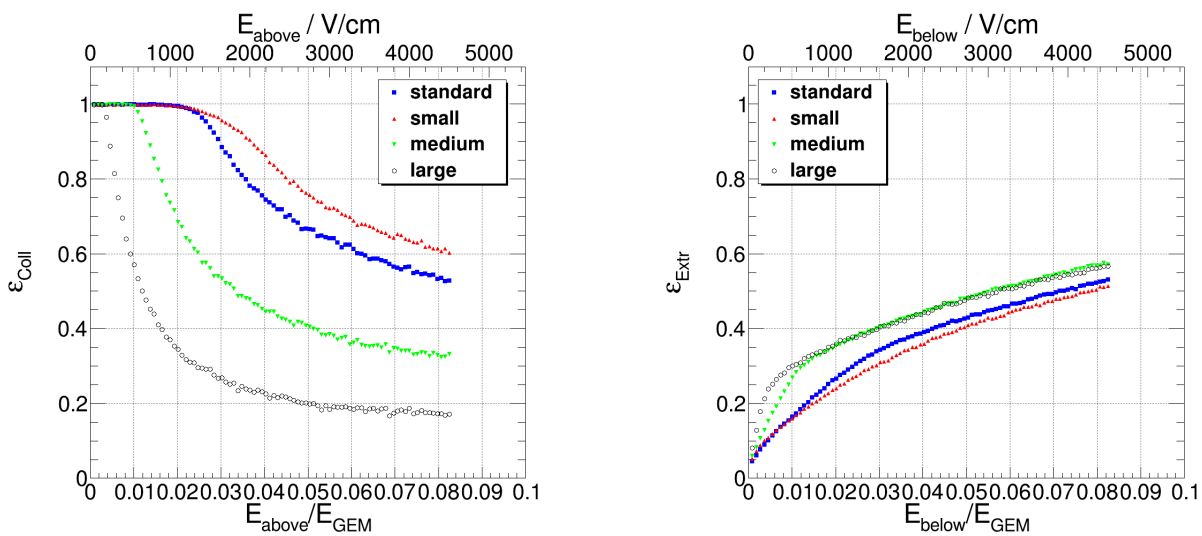

Figure 4: Garfield++ simulation of the collection and extraction efficiencies for different GEMgeometries.
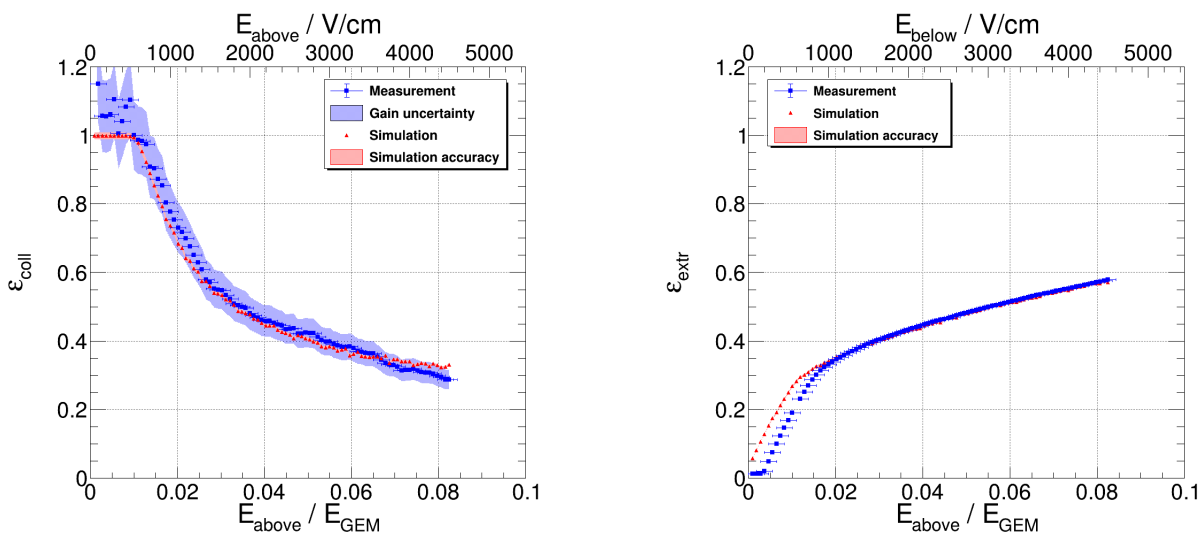

Figure 5: Comparison between Garfield++ simulation and measured collection and extraction efficiencies for a medium pitch GEM.

One way to prevent ions from drifting back into the sensitive area is to combine different GEM geometries [9]. Producing less ions in the beginning of the multiplication process while remaining the same effective gain corresponds to an improved electron transfer efficiency $\varepsilon$. Not all electrons are collected inside the holes of a GEM and extracted after multiplication. Some fraction will end up on the copper electrodes of the GEM, depending on the electrostatic configuration and the geometric parameters of the GEM. The GEM-types studied here are the so called standard $(140 \mu \mathrm{m})$, small $(90 \mu \mathrm{m})$, medium $(200 \mu \mathrm{m})$, and large $(280 \mu \mathrm{m})$ pitch GEMs, varying in the distance between the holes. Only the small pitch GEM has also different hole parameters than the other types. Here the outer diameter is $60 \mu \mathrm{m}$ and the inner diameter $40 \mu \mathrm{m}$ instead of $70 \mu \mathrm{m}$ and $50 \mu \mathrm{m}$ like for the other types. Simulated efficiencies to collect and extract electrons from a given GEM, as a function of the external fields, are shown in figure 4. They are in good agreement with measurements (see figure 5). 
While the absolute gain $G_{\text {abs }}$ of a single GEM only depends on the voltage difference which is applied over the GEM, the effective gain is reduced by these efficiencies: $G_{\text {eff }}=\varepsilon_{\text {Coll }} G_{\text {abs }} \varepsilon_{\text {Extr }}$. Increased transfer efficiencies of a GEM will result in a smaller required $G_{\text {abs }}$ and therefore a smaller voltage applied across the GEM. This leads to a more stable operation and reduces the number of ions flowing back into the drift volume.

While this treatment of a single GEM suggests values for the external fields, where the efficiencies will be maximised, the case of a stack composed of multiple GEMs is much more complicated, since the free parameters are coupled. The transfer field between two neighbouring GEMs will have an influence on the extraction efficiency of the one GEM and on the collection efficiency of the other. Inconveniently, the efficiencies show contrary dependence on the electric field. Still one can find a maximum for the transfer efficiency $\varepsilon_{\text {transfer }}=\varepsilon_{\text {extr }}^{i} \varepsilon_{\text {coll }}^{i+1}$ between two neighbouring GEMs, but this now depends on the geometry of the foils again.

\section{Summary and outlook}

The CBELSA/TAPS experiment will benefit substantially from the implementation of a TPC. A GEM-based prototype was comissioned and successfully operated, showing good results in terms of spatial, energy, and $\mathrm{d} E / \mathrm{d} x$ resolution. With the understanding of the transfer efficiencies of GEM foils with differen geometries, the optimisation of a whole stack of GEMs becomes possible.

\section{References}

[1] Sauli, F. (1997). GEM: A new concept for electron amplification in gas detectors. Nuclear Instruments and Methods in Physics Research Section A: Accelerators, Spectrometers, Detectors and Associated Equipment, 386 (2-3), 531-534.

[2] Thiel, A., et al. (2017). Double-polarization observable G in neutral-pion photoproduction off the proton. The European Physical Journal A, 53. Jg., Nr. 1, S. 8.

[3] Thiel, A., et al. (2012). Well-established nucleon resonances revisited by double-polarization measurements. Physical review letters, 109. Jg., Nr. 10, S. 102001.

[4] Gottschall, M., et al. (2014). First Measurement of the Helicity Asymmetry for $\gamma p \rightarrow p \pi^{0}$ in the Resonance Region. Physical review letters, 112. Jg., Nr. 1, S. 012003.

[5] Patrignani, C., et al. (2016). Review of particle physics. Chinese physics C, 40. Jg., Nr. 10, S. 100001.

[6] Baron, P., et al. (2008). AFTER, an ASIC for the readout of the large T2K time projection chambers. IEEE Transactions on Nuclear Science, 55. Jg., Nr. 3, S. 1744-1752.

[7] Berger, M., et al. (2017). A large ungated TPC with GEM amplification. Nuclear Instruments and Methods in Physics Research Section A: Accelerators, Spectrometers, Detectors and Associated Equipment, 869, 180-204.

[8] Böhmer, F., et al. (2014). First measurement of dE/dx with a GEM-based TPC. Nuclear Instruments and Methods in Physics Research Section A: Accelerators, Spectrometers, Detectors and Associated Equipment, 737. Jg., S. 214-221.

[9] Ottnad, J., et al. Charge-transfer properties of Gas Electron Multipliers. DPG Frühjahrstagung (DPG2017), Münster, Deutschland, March 27 - 312017. 